\title{
RESPONSE TO BORG AND BERGERMAIER
}

The comments by Borg and Bergermaier (1981) on our 'The structure of subjective well-being in nine western societies' (Andrews and Inglehart, 1979) raise a number of interesting methodological issues.

Particularly significant, we believe, is the notion that when only a small number of variables (there were 11 available to us) are mapped into a threedimensional space, there may be certain imprecisions in where those variables come to be placed: Equally good representations of the observed data may be achieved from modestly different structures. It follows that the nine countryspecific structures obtained in the analyses for our original article may not be precisely determined. There is no reason to believe, however, that the structures are not approximately correct; on the contrary, it seems unlikely that the modest indeterminacies in the placements of the variables would permit major revisions of the structures. Furthermore, these modest indeterminacies - and/or the use of measures of configurational similarity different from those we used - could indeed affect the relative similarities between structures obtained from different countries. In these respects, we believe the comments by Borg and Bergermaier represent a useful methodological addition to the results we reported.

When all is said and done, however, we believe the conclusion reached in our original article still obtains. This conclusions was that there exist "basic similarities between the structures of well-being assessments across the nine western societies examined..." (Andrews and Inglehart, 1979, p. 86). This finding seems important for comparative cross-national studies of perceived well-being, an enterprise that would be much more difficult if the very 'meaning' of well-being - as indicated by how aspects of well-being fit together in people's thinking - were found to differ radically from country to country. The high general level of similarity that we observed is the key empirical result of our analysis. The fact that the rank orders of these similarities might change somewhat if one were to use different similarity 
coefficients or because of minor imprecisions in locating the variables is irrelevant to our key result. Thus we strongly disagree with any implication that the Borg-Bergermaier analyses question the validity of our basic conclusion.

Our confidence in this conclusion was reinforced recently when we tried an alternative method - proposed to us by Ingwer Borg - for assessing the similarity between structures of well-being. Instead of first obtaining a spatial structure for each country by Smallest Space Analysis (with the attendant problem of imprecision noted above) and then assessing the similarities between these structures, as in our original analysis, Borg suggested that we simply compute the Pearson product-moment correlation between the corresponding elements in the correlation matrices for each pair of countries. We did that analysis, and the correlations among the 36 pairs of countries ranged from 0.66 to 0.88 (both mean and median were 0.79 ). Thus it is clear that there is a very substantial similarity in the pattern of relationships among the perceived well-being measures from one country to another. This simple descriptive analysis avoids the methodological issues raised by Borg and Bergermaier and confirms the conclusion we originally stated.

Borg and Bergermaier devote considerable attention to issues of statistical significance. Two observations need to be made. First, granting that none of the tests they cite seems ideal, all of the tests they report for our data indicate that the degree of similarity between the countries is statistically 'significant' beyond the conventional 0.05 probability level. In other words, by all of the tests assembled by Borg and Bergermaier, our conclusion is supported.

Second, in an insightful discussion of error types. Borg and Bergermaier observe (correctly) that as one becomes more 'conservative' with respect to making a Type I error, one increases the likelihood of making a Type II error. (In our case, a Type I error would be accepting the existence of a basic similarity between countries when there was no similarity, and a Type II error would be to conclude that no similarity exists when in fact it did.) We believe that Borg and Bergermaier have themselves fallen prey to this trap. In another set of comments on our article they wrote that our "finding of substantial agreement among the items' respective MDS representations has to be rejected" (Borg and Bergermaier, 1979, p. 253). Their statement is clearly at variance with the descriptive results presented above and seems a clear case of coming to a wrong conclusion (making a Type II error) while purporting to be 'conservative' with respect to a Type I error. In a world of real issues 
and imperfect measures and methodologies, statistical sophistication needs to be combined with practical sense. Given the data analyzed in our article (which are unusually extensive, but still limited and imperfect), all indications support the hypothesis that there do exist basic similarities among the nine countries in the way people structure their perceptions of well-being.

\section{University of Michigan}

\section{BIB LIOG R A PH Y}

Andrews, F. M. and Inglehart, R. F.: 1979, The structure of subjective well-being in nine western societies', Social Indicators Research 6, pp. 73-90.

Borg, I. and Bergermaier, R.: 1979, 'Die Ähnlichkeit von Einstellungsstrukturen zur Lebensqualität in elf westlichen Gesellschaften', Zeitschrift für Sozialpsychologie 10 , pp. 253-261.

Borg, I. and Bergermaier, R.: 1981, 'Some comments on "The structure of subjective well-being in nine western societies" by Andrews and Inglehart', Social Indicators Research 9, this issue, pp. $265 f$. 